\title{
Retrospective review using targeted deep sequencing reveals mutational differences between gastroesophageal junction and gastric carcinomas
}

Hector H Li-Chang ${ }^{1,2,3^{*}}$, Katayoon Kasaian ${ }^{1,4}$, Ying Ng ${ }^{5}$, Amy Lum5 ${ }^{5}$, Esther Kong ${ }^{5}$, Howard Lim ${ }^{1,6}$, Steven JM Jones ${ }^{4}$, David G Huntsman ${ }^{1,2,3,5}$, David F Schaeffer ${ }^{1,2}$ and Stephen Yip ${ }^{1,2,5}$

\begin{abstract}
Background: Adenocarcinomas of both the gastroesophageal junction and stomach are molecularly complex, but differ with respect to epidemiology, etiology and survival. There are few data directly comparing the frequencies of single nucleotide mutations in cancer-related genes between the two sites. Sequencing of targeted gene panels may be useful in uncovering multiple genomic aberrations using a single test.

Methods: DNA from 92 gastroesophageal junction and 75 gastric adenocarcinoma resection specimens was extracted from formalin-fixed paraffin-embedded tissue. Targeted deep sequencing of 46 cancer-related genes was performed through emulsion PCR followed by semiconductor-based sequencing. Gastroesophageal junction and gastric carcinomas were contrasted with respect to mutational profiles, immunohistochemistry and in situ hybridization, as well as corresponding clinicopathologic data.

Results: Gastroesophageal junction carcinomas were associated with younger age, more frequent intestinal-type histology, more frequent p53 overexpression, and worse disease-free survival on multivariable analysis. Among all cases, 145 mutations were detected in 31 genes. TP53 mutations were the most common abnormality detected, and were more common in gastroesophageal junction carcinomas ( $42 \%$ vs. $27 \%, p=0.036$ ). Mutations in the Wnt pathway components APC and CTNNB1 were more common among gastric carcinomas ( $16 \% \mathrm{vs.} 3 \%, \mathrm{p}=0.006$ ), and gastric carcinomas were more likely to have $\geq 3$ driver mutations detected ( $11 \% \mathrm{vs}$. $2 \%, \mathrm{p}=0.044$ ). Twenty percent of cases had potentially actionable mutations identified. R132H and R132C missense mutations in the IDH1 gene were observed, and are the first reported mutations of their kind in gastric carcinoma.

Conclusions: Panel sequencing of routine pathology material can yield mutational information on several driver genes, including some for which targeted therapies are available. Differing rates of mutations and clinicopathologic differences support a distinction between adenocarcinomas that arise in the gastroesophageal junction and those that arise in the stomach proper.
\end{abstract}

Keywords: Gastric cancer, Gastroesophageal junction cancer, Gastric cancer genomics, Gastric cancer sequencing

\footnotetext{
* Correspondence: hlichang@bccrc.ca

${ }^{1}$ University of British Columbia, Vancouver, Canada

${ }^{2}$ Division of Anatomic Pathology, Department of Pathology and Laboratory

Medicine, Vancouver General Hospital, 85512 Ave W, Vancouver, BC V5Z 1 M9,

Canada

Full list of author information is available at the end of the article
} 


\section{Background}

Gastric cancer accounts for over 10,000 deaths annually in the United States [1], and is the second most common cause of cancer mortality worldwide [2]. Although carcinomas of the gastroesophageal junction (GEJ) have been grouped with gastric carcinomas in cancer registries and in clinical trials for targeted therapies [3], lesions at these two sites have distinct clinical features. Adenocarcinomas of the stomach proper are primarily caused by Helicobacter pylori infection [4] and are decreasing in incidence worldwide [1]. In contrast, GEJ cancers are most associated with gastroesophageal reflux disease [2-5] and obesity [6], and the incidence of GEJ carcinomas has remained stable over the past 20 years [7]. In addition, the prognosis of GEJ carcinomas has been noted to be worse than gastric carcinomas, and there is uncertainty as to whether GEJ carcinomas should be staged as gastric or esophageal tumors [8]. Recognizing the distinction between carcinomas of the GEJ, esophagus, and stomach may enhance the collection of meaningful epidemiologic data and result in increased management precision [9].

Several studies have noted differences in the molecular characteristics of GEJ carcinomas versus those that arise elsewhere in the stomach. TP53 mutations are more frequent in the GEJ than in the distal stomach, while loss of heterozygosity of the TP53 locus is also more common in GEJ tumors $[10,11]$. Significant differences in promoter methylation rates of $A P C$ and $C D K N 2 A$ have also been described [12]. Furthermore, differences in $A P C$ mutation rates and protein expression, as well as differences in global gene expression profiles between the two sites have also been demonstrated [13-16].

Testing of amplifications of the ERBB2 (also known as $H E R 2)$ gene in gastric and gastroesophageal junction cancers is now routine practice in many institutions [17]. Similarly, testing for driver mutations, particularly single nucleotide substitutions, in oncogenes and tumour suppressor genes currently informs treatment in adenocarcinomas of other sites such as the lung and colon [18-20]. As further molecular targets are discovered across disease sites, effective assays will be required to determine cancers' susceptibility to targeted treatment.

Next-generation sequencing may be used in the near future to interrogate multiple genes in a single sample, and these data could be used to inform clinicians of driver mutations and guide targeted treatment. Targeted panel sequencing is a form of next-generation sequencing where single nucleotide variants are detected in a limited number of previously determined genomic loci, which by intention are often prognostically and therapeutically critical. Panel sequencing enables multiplexing of samples, and deep coverage $(>500 \mathrm{x})$ facilitates the analysis of suboptimal template material from archival tissue and samples with low tumor cellularity. The narrower set of genes also allows for quicker specimen processing and bioinformatic analysis. Thus, actionable results can be obtained within days, rather than the weeks, compared to whole genome and exome approaches. However, data is restricted by the inherently biased selection of genes, and the inability to detect copy number changes, loss of heterozygosity, and structural rearrangements such as gene fusions. Thus, the effective use of NGS requires careful assessment of technologies, assay limitations, template requirements, and the research and clinical questions under consideration.

The objectives of this study were to probe the utility of panel sequencing on formalin-fixed paraffin-embedded (FFPE) tissue, and to compare clinically annotated GEJ and gastric carcinomas through panel sequencing of the hotspots of 46 cancer genes. We also sought to compare the frequencies of mutations identified with panel sequencing of hotspots against whole-exome sequencing, using publically available data from The Cancer Genome Atlas.

\section{Methods}

\section{Case selection and retrieval of clinicopathologic data}

Institutional ethics approval was obtained from the University of British Columbia/British Columbia Cancer Agency research ethics board (\#H07-2807), and research was conducted in accordance with the Helsinki declaration. Cases of gastric carcinoma were retrieved from departmental archives from the British Columbia Cancer Agency (BCCA), a provincial referral center. Inclusion criteria were referral to the agency between 2004 and 2010, available FFPE tissue from surgical resection of the primary tumor, complete clinicopathologic data including clinical outcomes on follow-up, and the absence of metastatic disease at presentation. Biopsy specimens of primary and metastatic lesions were excluded due to the absence of complete pathologic data. GEJ location was defined as lesions with an epicenter within $5 \mathrm{~cm}$ of the proximal end of the gastric rugal folds [21]. No distinction was made between tumors with regards to the location of their epicenter within the $5 \mathrm{~cm}$ of the GEJ (i.e. Siewert type was not recorded) [22]. Carcinomas located exclusively within the esophagus were excluded, as per the most recent WHO criteria [21]. All gastric tumors located distal to the GEJ were binned together for this study. Clinicopathologic data was collected retrospectively through review of patients' charts by a member of the clinical team, as well as through review of pathology reports.

\section{Tissue microarray construction, immunohistochemistry and in situ hybridization}

Tissue microarray construction was carried out using two $0.6 \mathrm{~mm}$ cores from two separate sections of tumor. Immunohistochemical staining for p53 (1:100; clone 
DO-7, Ventana Medical Systems, Tucson, AZ), Baf250a (1:75; Sigma-Aldrich, St. Louis, MO), and the mismatch repair (MMR) proteins including hMLH1 (1:25; clone ES05, Leica, Wetzlar, Germany), MSH2 (1:5; clone 25D12, Leica), hMSH6 (1:300; clone PU29, Leica), and hPMS2 (1:150; clone MOR4G, Leica) was performed on the XT platform (Ventana). Expression of p53 was scored as absent $(<1 \%$ nuclear staining), normal (1-60\% nuclear staining of any intensity), or overexpression ( $>60 \%$ nuclear staining of any intensity). Baf250a and MMR proteins were scored as intact ( $\geq 1 \%$ staining) or negative $(<1 \%$ staining) based on protein expression specifically in tumour cells (i.e. immune and stromal expression was ignored). ERBB2 silver in situ hybridization (SISH) was performed using the XT automatic IHC/ISH staining platform (Ventana). A $E R B B 2: C E P 17$ ratio $<2.0$ was classified as non-amplified, and a value $\geq 2.0$ as amplified. Enumeration of SISH signals was based on established protocols [17].

\section{DNA sample processing, sequencing, and variant calling}

In each case, hematoxylin and eosin slides were used to guide macrodissection or scrolling of tumor tissue from FFPE slides following outlining of tumours by an anatomical pathologist. Tumor DNA from each case was extracted using Qiagen FFPE DNA extraction kit (Qiagen, Venlo, Netherlands); no germline DNA was extracted. Extracted DNA was quantified using the QUBIT HS dsDNA assay (Life Technologies Gaithersburg, MD, USA); all cases had a minimum of 10 ng of DNA extracted from FFPE, in keeping with a previously reported requirement for the assay [21]. A minimum A260/280 ratio of 1.8 was required for each DNA sample. DNA amplicon library construction was performed using DNA primers from the Ion Ampliseq ${ }^{\text {wi }}$ Cancer Hotspot Panel v1 (Life Technologies). The kit consists of 207 primer pairs that cover 739 hotspots within 46 cancer-related genes (Additional file 1: Table S1). Indexed amplicon libraries were pooled for emulsion polymerase chain reaction and sequencing on the Ion Torrent PGM platform (Life Technologies). A minimum of at least $500 x$ base pair coverage was required for each case. Variant calling was performed using the Torrent Variant Caller v2.2 (Life Technologies) using the hg19 reference genome. Only variants present at frequencies $\geq 5 \%$ were considered. Because germline DNA was unavailable for comparison, variants were excluded as possible somatic mutations if they were identified as single nucleotide polymorphisms with mean allele frequencies of $>0$ within the dbSNP database (www.ncbi.nlm.nih.gov/SNP); their status as nongermline variants was further confirmed using a PubMed search (www.ncbi.nlm.nih.gov/pubmed).

Comparison with the Cancer Genome Atlas (TCGA) data Curated somatic mutation calls for 281 TCGA stomach adenocarcinoma samples with known anatomical sites were retrieved from the TCGA Data Portal (https://tcgadata.nci.nih.gov/tcga/) on February 19, 2014. Protein-coding mutations located in the regions amplified by the Ion Ampliseq $^{\text {min }}$ Cancer Hotspot Panel v1 in each of the 46 genes were obtained for cases and stratified by location (60 cardia/proximal and gastroesophageal junction versus 221 fundus/body, antrum/distal and stomach NOS). Copy number data, RNA expression data, and protein expression data were not considered as our own assay only detects single nucleotide variants (SNVs) and small basepair insertions/deletions (INDELs). The frequencies of mutations, irrespective of the type of mutation, were compared versus the hotspot multiple panel sequencing that we performed.

\section{Data analysis}

Mann-Whitney U-tests and student t-tests were used to compare linear variables, where appropriate. Fisher exact and chi-square tests, where appropriate, were used to compare categorical values. Survival analyses were performed using log-rank (Kaplan-Meier) and Cox proportional hazards tests. The 46 panel genes were mapped to the Kyoto Encyclopedia of Genes and Genomes (KEGG) [22,23] and the Ingenuity ${ }^{\circ}$ Integrated Pathway Analysis program (Qiagen) to identify oncogenic pathways and networks enriched for mutations, and to test for statistically significant differences between gastroesophageal junction and gastric adenocarcinoma specimens. $P$ values were corrected for multiple testing using the Benjamini-Hochberg (BH) correction [24]. All statistical tests were two-tailed and a $P$ value of $<.05$ was considered statistically significant. Statistical analyses were performed using SPSS Statistics software (v22, IBM, Armonk, NJ, USA) and the R statistical language v.2.15.1 (R Core Team (2012). R: A language and environment for statistical computing. $R$ Foundation for Statistical Computing, Vienna, Austria. ISBN 3-900051-07-0, URL http://www.R-project.org/).

\section{Results}

Within departmental archives at the BCCA, 229 resection specimens of gastric and GEJ carcinomas were obtained from 2004 to 2010 and were available for construction of a tissue microarray. DNA was available for extraction for 176 cases. No clinicopathologic data was available for correlation in 6 cases. Three cases had metastatic disease documented within a month of presentation, and these were excluded from the analysis. Of the remaining 167 cases, 92 originated in the gastroesophageal junction and 75 originated in the remainder of the stomach (Figure 1).

\section{Clinicopathologic differences between GEJ and gastric carcinomas}

The clinicopathologic features of these cases are summarized in Table 1 and anonymized clinical data is provided 


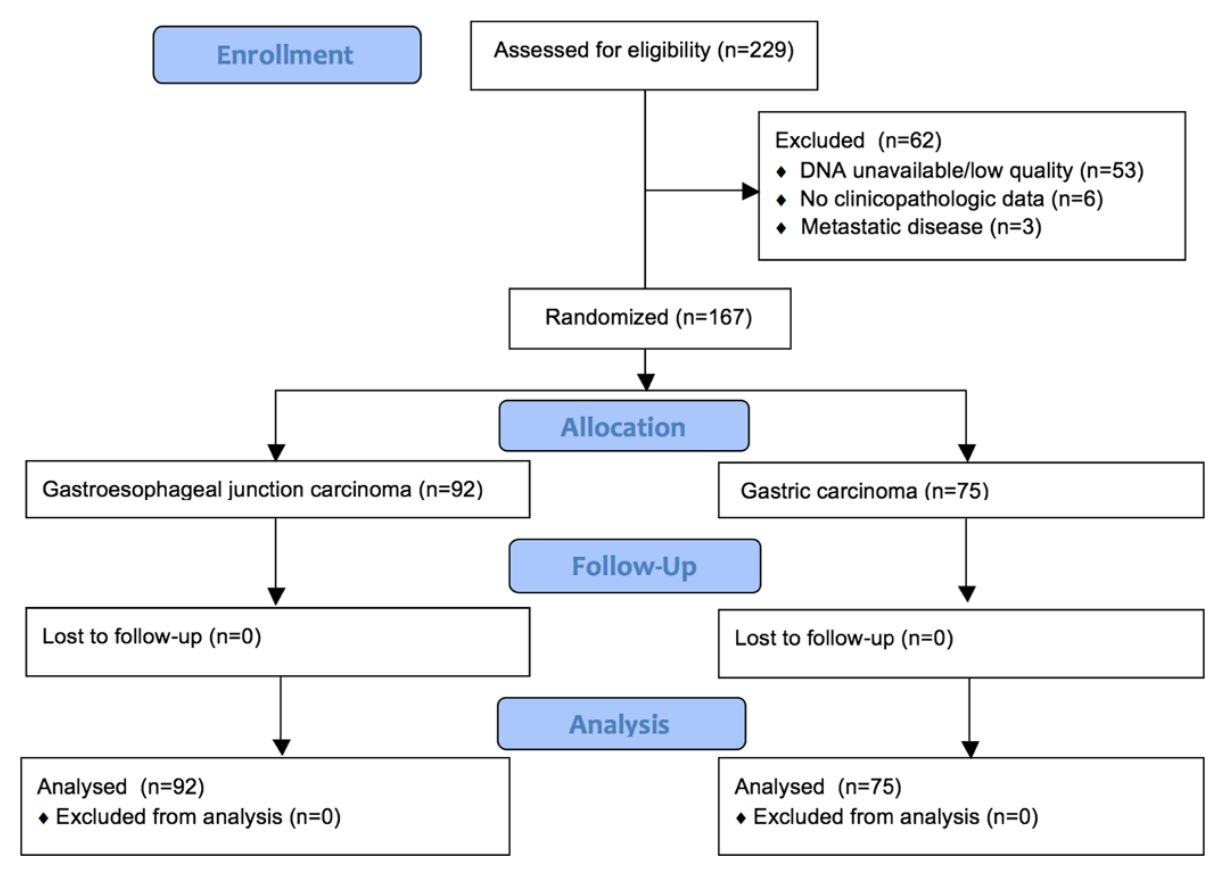

Figure 1 Flow diagram detailing case selection and exclusion for the study cohort.

in a supplemental file (Additional file 2: Table S2). GEJ carcinomas were associated with younger age at resection, more frequent intestinal-type and less frequent diffuse histology, more frequent p53 overexpression and less frequent loss of p53 expression, more frequent stage III disease, less frequent stage I disease, and more frequent recurrences. Disease-free survival was significantly worse among patients with GEJ carcinomas (Figure 2A), though the two cohorts were not statistically different in terms of overall survival (Figure 2B). Other clinicopathologic features were similar between tumors of the two locations, including $\mathrm{T}$-stage, resection margin involvement, ERBB2 amplification, and MMR protein loss (Table 1). The proportion of diffuse carcinomas in the Lauren classification) was similar between the two sites. Subgroup analysis of only intestinal-type carcinomas showed persistent differences between GEJ and gastric carcinomas in disease-free survival and p53 expression. Differences in age, p53 expression and outcome persisted when considering only intestinal-type carcinomas, as well as when tumours were stratified into three subtypes (proximal nondiffuse, diffuse, and distal non-diffuse) as suggested by Shah et al. [16] (Additional file 3: Table S3).

On multivariable analysis, GEJ location was independently associated with worse disease-free survival (Cox Proportional Hazard $=2.08$ [95\% confidence interval: 1.25-3.44], $\mathrm{p}=0.005$ ) along with margin status and microsatellite instability (Additional file 4: Table S4). Age, tumor grade and margin involvement were independently prognostic of overall survival (Additional file 5: Table S5).

\section{Mutations identified with the cancer panel}

Among all cases, 145 mutations were detected in 31 genes, with 75 mutations detected among 57 of the tumors from the GEJ, and 70 mutations detected among 43 gastric tumors (Figure 3). No mutations were detected in 35 (38\%) and $32(43 \%)$ of the tumors from the GEJ and stomach, respectively. TP53 was the most commonly mutated genes, with variants identified in 59 of 167 cases (35\%). The next most commonly mutated genes were PI3KCA (6\%), CTNNB1 (5\%), KRAS (5\%) and SMAD4 (4\%). Other variants included hotspot mutations in IDH1 (2 cases), JAK3 (3 cases), and FLT3 (2 cases). A single mutation was identified in 70 cases (42\%), 2 mutations were identified in 20 cases $(12 \%)$, and $\geq 3$ mutations were identified in 10 cases $(6 \%)$.

No mutations were identified within the hotspot regions of ALK, CSF1R, EGFR, FGFR2, HNF1A, HRAS, JAK2, $M P L, N P M 1, N R A S, S R C, S T K 11$ or VHL. All variant calls are available in the supplementary data (Additional file 6: Table S6).

\section{Differences in mutations between the GEJ and stomach} TP53 mutations were identified in 39 of 92 (42\%) of GEJ tumors, and in 20 of $75(27 \%)$ gastric tumors $(\mathrm{p}=0.036)$. When subdivided into the 3 subtypes suggested by Shah et al. [16], TP53 mutations occurred more frequently in proximal nondiffuse cancers $(44 \%)$ than in diffuse cancers (37\%) and distal nondiffuse cancers $(20 \% ; \mathrm{p}=0.024)$. This classification also showed more frequent mutations in KRAS within distal nondiffuse cancers (12\%) versus 
Table 1 Summary of the clinocopathologic variables in the cohort's clinicopathologic variables within cardia and non-cardia adenocarcinomas

\begin{tabular}{|c|c|c|c|c|}
\hline Clinicopathologic variable & Gastroesophageal junction $(n=92)$ & Non-cardia $(n=75)$ & Overall $(n=167)$ & $p$ \\
\hline Age (mean, years) & $61.5+/-9.6[33-80]$ & $66.3+/-11.9[33-84]$ & $63.7+/-10.9$ [33-84] & 0.001 \\
\hline Sex & & & & 0.297 \\
\hline Male & $70(76)$ & $51(68)$ & $121(73)$ & \\
\hline Female & $22(24)$ & $24(32)$ & $46(27)$ & \\
\hline Histologic subtype (Lauren) & & & & 0.008 \\
\hline Intestinal & $65(71)$ & $36(48)$ & $101(60)$ & \\
\hline Diffuse & $15(16)$ & $26(35)$ & $41(25)$ & \\
\hline Mixed & $12(13)$ & $13(17)$ & $25(15)$ & \\
\hline Stage (AJCC) & & & & 0.031 \\
\hline $\mid A-B$ & $10(11)$ & $19(25)$ & $29(17)$ & \\
\hline$\| A-B$ & $60(65)$ & $45(60)$ & $105(63)$ & \\
\hline$\| 1 \mid-A-C$ & $22(24)$ & $11(15)$ & $33(20)$ & \\
\hline Grade & & & & 0.415 \\
\hline Well differentiated (G1) & & $4(5)$ & $10(6)$ & \\
\hline Moderately differentiated (G2) & $39(42)$ & $25(33)$ & $64(38)$ & \\
\hline Poorly differentiated (G3) & $47(51)$ & $46(61)$ & $93(56)$ & \\
\hline Resection margin & & & & 0.306 \\
\hline Uninvolved & $74(80)$ & $65(87)$ & $139(83)$ & \\
\hline Involved & $18(20)$ & $10(13)$ & $28(17)$ & \\
\hline ERBB2 amplification & & & & 0.654 \\
\hline Absent & $78(85)$ & $66(88)$ & $144(86)$ & \\
\hline Present & $14(15)$ & $9(12)$ & $23(14)$ & \\
\hline BAF250a (ARID1A) expression & & & & 0.111 \\
\hline Intact & $73(79)$ & $51(68)$ & $124(74)$ & \\
\hline Absent & $19(21)$ & $24(32)$ & $43(26)$ & \\
\hline p53 expression & & & & $2.8 \times 10^{-4}$ \\
\hline 0 - absent & $32(35)$ & $47(63)$ & $79(47)$ & \\
\hline 1 - normal (1-60\%) & $17(19)$ & $14(19)$ & $31(19)$ & \\
\hline 2 - increased (>60\%) & $43(47)$ & $14(19)$ & $57(34)$ & \\
\hline Mismatch repair proteins & & & & 0.244 \\
\hline Intact & $77(84)$ & $57(76)$ & $135(80)$ & \\
\hline Abnormal & $15(16)$ & $18(24)$ & $33(20)$ & \\
\hline Number of recurrences & $57(62)$ & $32(43)$ & $89(53)$ & 0.019 \\
\hline Median progression-free survival (Months) & 12 & 18 & 15 & \\
\hline Number of deaths & $69(75)$ & $48(64)$ & $117(70)$ & 0.130 \\
\hline Median overall survival (Months) & 18.0 & 23.0 & 20.0 & \\
\hline
\end{tabular}

proximal nondiffuse $(3 \%)$ and diffuse $(0 \%)$ carcinomas $(\mathrm{p}=0.12)$. No significant differences in mutation frequencies were present among the other individual genes in the panel. Two components of the Wnt pathway, APC and CTNNB1, were in aggregate mutated more frequently in gastric carcinomas than in GEJ tumors (16\% vs. $3 \%$, $\mathrm{p}=0.006)$. Gastric carcinomas more frequently had mutations in 3 or more genes ( $11 \%$ vs. $2 \%, \mathrm{p}=0.044$; Figure 4$)$.
No differences in the involvement of oncogenic pathways were noted between the two sites, based on mutational profiles.

\section{Potentially actionable mutations}

Targeted therapies are available or in development for mutations occurring in the following genes: $A K T$ [25], $B R A F$ [26], ERBB2 [27], ERBB4 [28], FGFR1 [29], FGFR3 


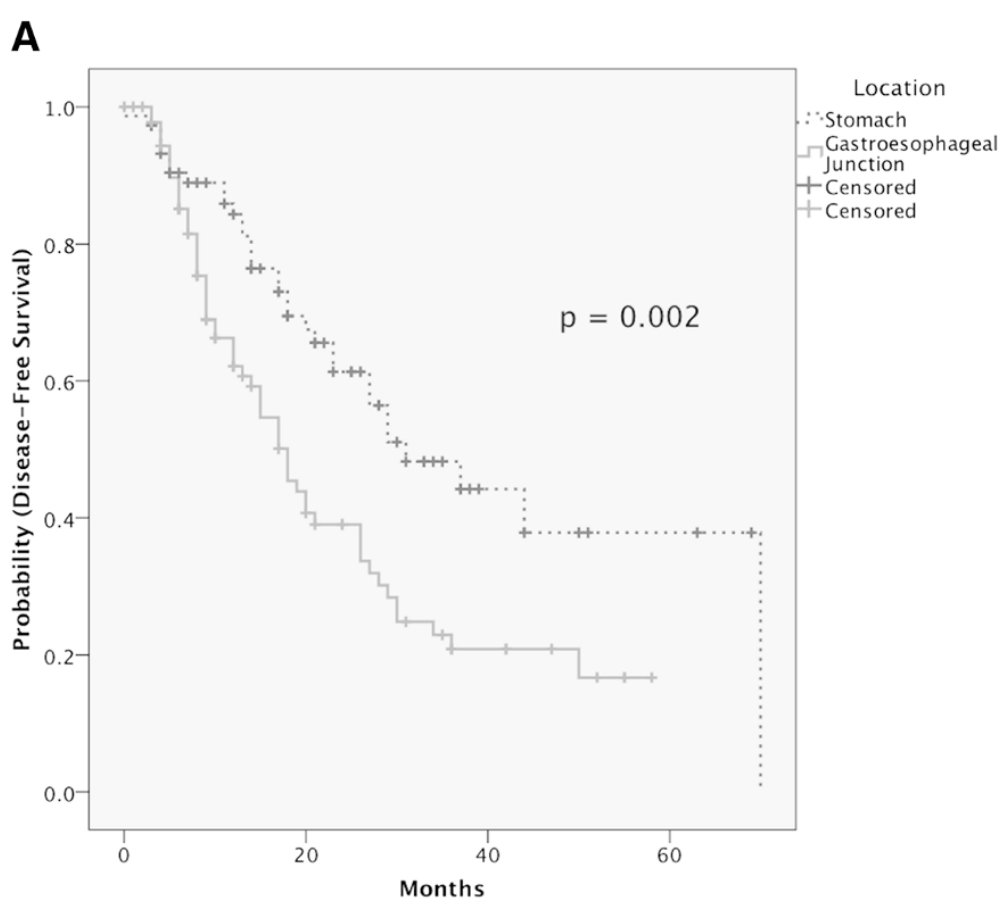

B

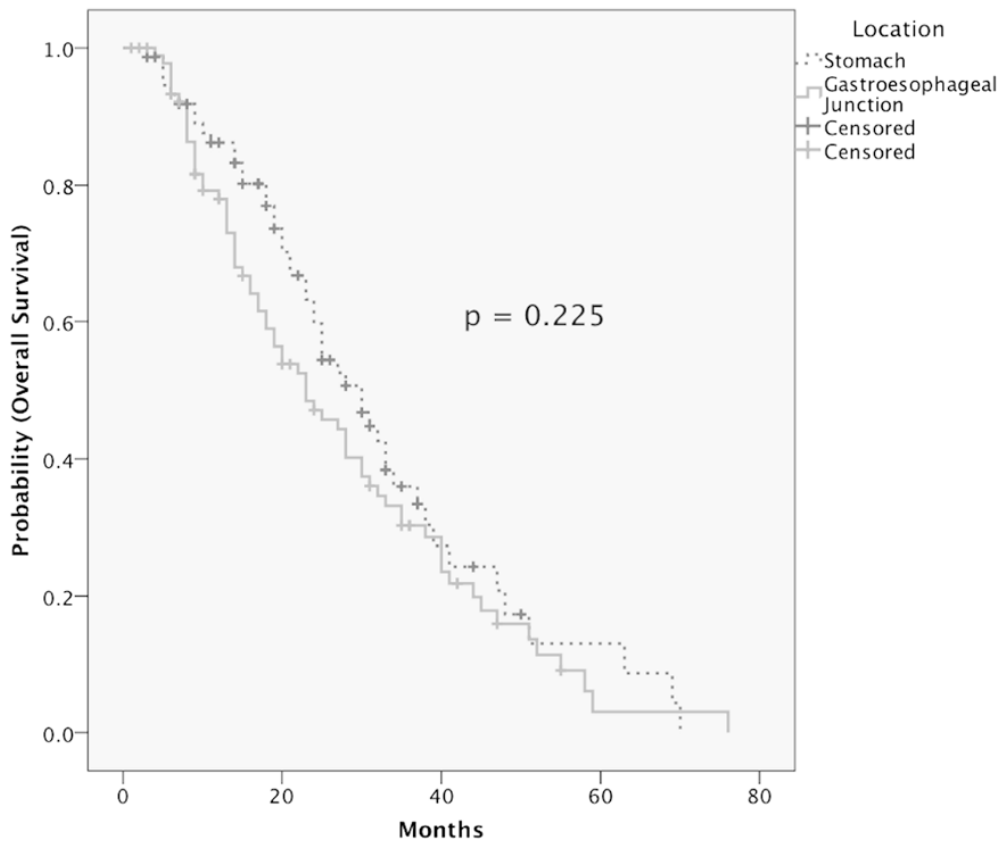

Figure 2 Comparison of disease-free survival and overall survival between patients with gastroesophageal and gastric carcinomas.

A) Disease free survival was significantly worse for gastroesophageal carcinomas (solid lines) compared to gastric carcinoms (dotted lines), Log-rank test; $p=0.002$, though $\mathbf{B}$ ) overall survival did not differ between the two disease sites (Log-rank test; $p=0.225$ ).

[30], FLT3 [31,32], IDH1 [33], JAK3 [31], KDR [34,35], KRAS [36], MET [34], PDGFRA [37], PIK3CA [25], PTEN [25], PTPN11 [38], RET [39], SMO [40]. Mutations in these genes were identified in 32 cases (19\%), including 6 cases (4\%) with 2 mutations and 3 cases (2\%) with $\geq 3 \mathrm{mu}-$ tations. The distribution of actionable mutations was not significantly different between GEJ and gastric carcinomas $(\mathrm{p}=0.327$; Figure 4$)$.

\section{Prognostic significance of mutations}

$E R B B 4$ mutations were associated with worse diseasefree survival $(\mathrm{p}=0.018)$, while there was a trend towards 


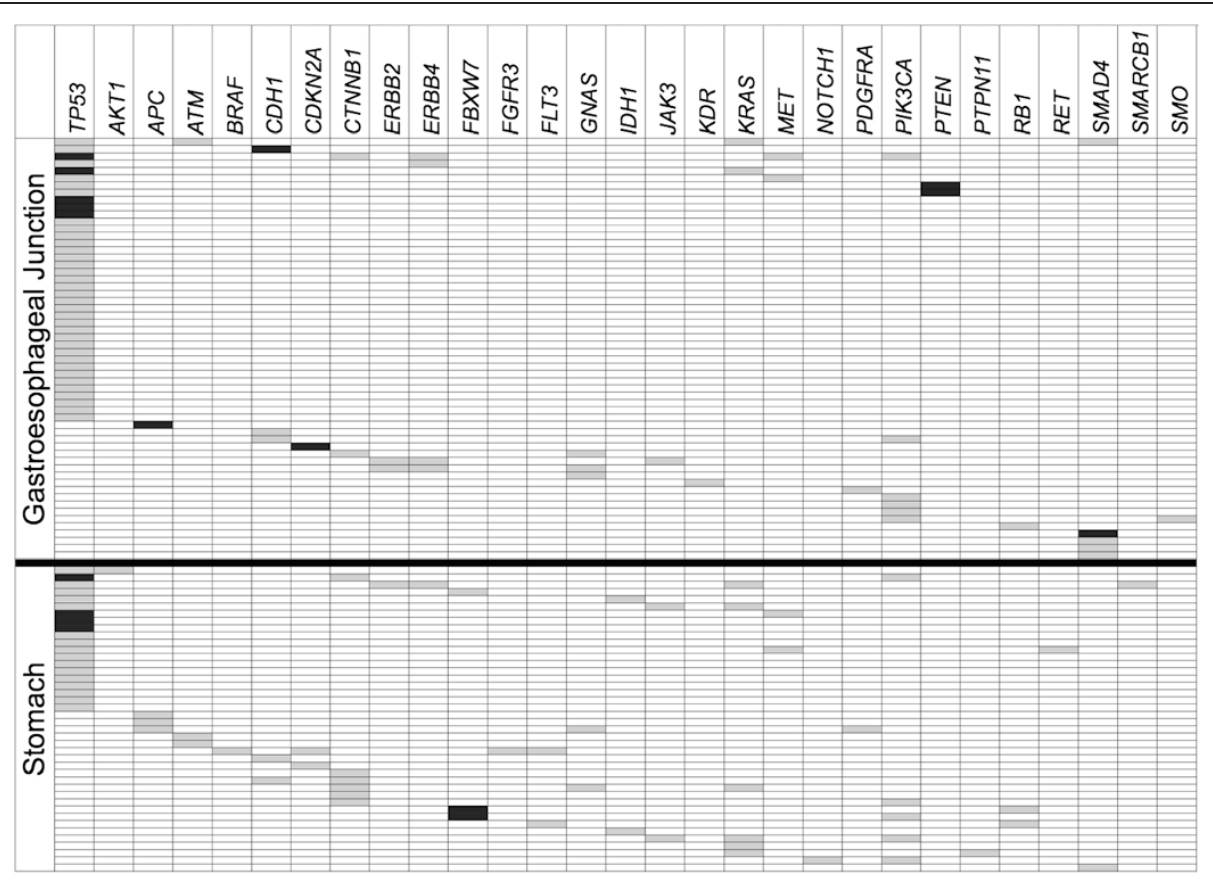

Figure 3 Somatic mutations identified in gastroesophageal junction and gastric carcinomas. TP53 mutations were identified in a larger proportion of gastroesophageal junction tumors, while abnormalities in APC/CTNNB1 occurred more frequently in gastric tumors. Black blocks represent truncating mutations, while grey blocks represent missense mutations. Cases and genes in which mutations were not identified are not included.

worse disease-free survival associated with mutations in $A B L 1 \quad(\mathrm{p}=0.063)$ and JAK3 $(\mathrm{p}=0.059)$. None of these mutations were prognostically significant after accounting for age, sex, Lauren subtype, stage, grade and margin status. Mutations in BRAF ( $\mathrm{p}<0.001)$, FGFR3 ( $\mathrm{p}<0.001)$, FLT3 $(\mathrm{p}<0.001)$ were associated with worse overall survival

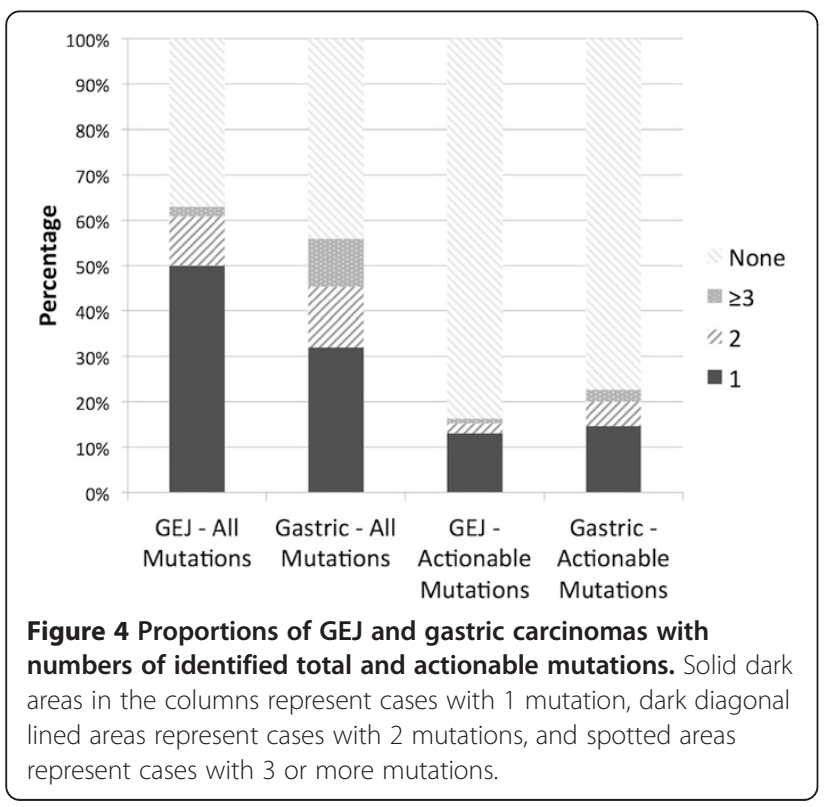

on univariate analysis as a result of a single case with mutations in all three of these genes.). BRAF mutation remained prognostically significant after accounting for age, sex, Lauren subtype, stage, grade and margin status $(\mathrm{p}=0.002)$.

\section{Comparison with TCGA data}

When assessing the hotspot regions covered by the sequencing panel, the overall number of mutated genes per case was similar between the TCGA and study cohorts $(\mathrm{p}=0.659)$, including when comparing either GEJ $(\mathrm{p}=$ $0.399)$ or gastric $(p=0.845)$ tumors only (Figure $5 \mathrm{~A})$. A trend towards more frequent cases with mutations in $\geq 3$ genes in the stomach compared to the GEJ was also observed in the TCGA data $(12 \%$ vs. $3 \%, \mathrm{p}=0.054)$. The overall frequency of TP53 mutations was not different between the study cohort and the TCGA cohort $(\mathrm{p}=0.230)$. No differences in TP53, KRAS, and APC/CTNNB1 mutation rates between GEJ and gastric carcinomas were observed in the TCGA dataset (Figures 5B-D). The mutated genes in the TCGA data set are included in Additional file 7: Table S7. Regarding the mutations with possible prognostic significance identified in our cohort, there was a trend towards worse overall survival associated with BRAF mutations $(\mathrm{p}=0.079)$, while no prognostic association was found in the TCGA cohort in association with mutations in ERBB4, ABL1, JAK3, FLT3 or FGFR3. 


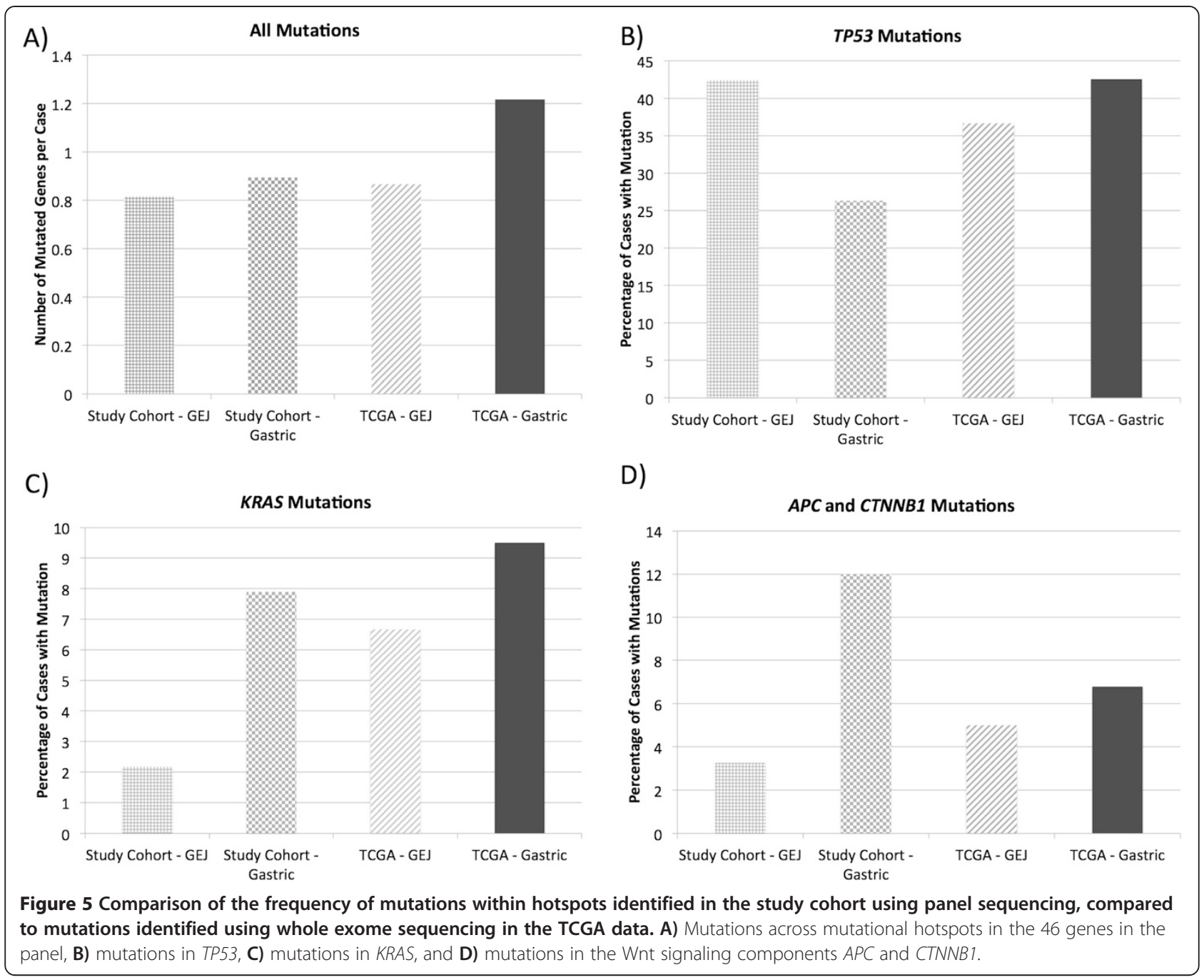

\section{Discussion}

This study aimed to probe the utility of panel sequencing in identifying single nucleotide changes in routinely processed gastric resection specimens, which could be used to guide targeted therapies. We secondarily sought to contrast GEJ and gastric carcinomas through targeted deep sequencing of a panel of 46 cancer-related genes, which revealed some differences at the genomic level that may reflect differing clinicopathologic profiles. Finally, we also sought to compare the frequencies of mutations obtained using this panel with results from whole exome sequencing in The Cancer Genome Atlas.

Adenocarcinomas of the gastrointestinal tract are molecularly heterogeneous and complex [41-44]. In gastric carcinoma, deep sequencing of single nucleotide polymorphism and RNA expression arrays have recently revealed abnormalities in several pathways including WNT, Hedgehog, cell cycling, DNA damage repair and the epithelial-to-mesenchymal transition [45]. The current use of multiple single gene tests is untenable given this complexity, particularly in the presence of a growing number of targeted therapies, constrained resources, and limited tissue availability. Thus, it is desirable to investigate multiple genes simultaneously. Panel sequencing has a sensitivity of close to $100 \%$ relative to conventional assays such as Sanger sequencing and PCR-based methods, as well as an ability to detect SNVs and INDELs at allele frequencies as low as 5\% and 20\%, respectively, in both FFPE [21,46-48] and cytology specimens [49-52]. Targeted panel sequencing can detect aberrations in cancer-related genes in early gastric cancers and precursors lesions [53], and its deep coverage could be particularly useful in gastric cancer by providing adequate results despite scant biopsy material and the admixture of tumor cells with desmoplasia and inflammatory cells.

Putative driver mutations were identified in a majority of GEJ and gastric carcinomas investigated in this study. By far the most frequently detected mutated gene was TP53, and these mutations have also been detected in early stage and precursor lesions using the same assay 
[53]. Multiple driver mutations were identified in several cases, reinforcing the idea that multiple genes need to be interrogated at once in genomically complex tumors such as gastric adenocarcinomas. A case with a mutation in $B R A F$ (as well as FLT3 and FGFR3) was associated with poor overall survival on both univariate and multivariable analysis. This finding mirrors a trend observed in the TCGA data towards poor overall survival in BRAFmutated tumours, suggesting that in some cases panel sequencing could have a prognostic role.

We were also able to detect potentially actionable mutations in approximately $20 \%$ of cases, which involved either genes or pathways where targeted therapies are available or in development. While this number would ideally be higher, our assay only covered certain hotspot regions of these genes, and did not account for copy number alterations that could also yield useful information. Further refinement of such panels to include a broader range of genes and gene segments will likely increase the proportion of cases in which mutations are identified. For example, although TP53 mutations occur throughout the gene, the panel primarily covers exons $5-8$, and some of the gene segments that were not sequenced are more frequently associated with loss of p53 on immunohistochemistry [54]. This fact may potentially explain both the differences in the rates of TP53 mutations and patterns of immunohistochemical expression observed in the GEJ and stomach. Nevertheless, this study does demonstrate that single nucleotide variants can be identified from routine/archival pathology materials, and that with additional refinement panel sequencing may have a significant role in the future.

An unexpected result of the cancer hotspot panel sequencing approach was the identification of mutations in genes usually associated with non-epithelial malignancies, such as IDH1 R132H/R132C, JAK3 V722I, and FLT3 A680V. The IDH1 variants identified occur primarily in glial and hematologic malignancies, and result in altered cancer cell metabolism [55]. To the best of our knowledge, these cases constitute the first report of pathogenic IDH1 mutations in gastric cancer. Recently IDH1 mutations have been targeted [33], and mutationspecific treatments are currently the aim of a phase I clinical trial that includes cholantiocarcinomas (http:// clinicaltrial.gov/ct2/show/NCT02073994). FLT3 mutations occur in a third of cases of acute myelogenous leukemia [56], and the point mutation resulting in the A680V substitution has not been previously described in gastric cancer, while being observed occasionally in AML [57]. Similarly, activating JAK3 mutations such as V722I have been identified in acute megakaryoblastic leukemia [58] and NK/T-cell lymphoma [31], and only in a few cases of gastric and breast cancer [59].
Epidemiologic and clinicopathologic differences exist between GEJ and gastric carcinomas [60-62]. GEJ carcinomas in this cohort were associated with younger age, different histotypes, and worse disease-free survival. As in other series, the rates of p53 overexpression were higher in the GEJ, as were the rates of TP53 mutation [10,11], while Wnt abnormalities were more common in the gastric carcinomas [12]. In addition, more frequently there were mutations across $\geq 3$ genes in gastric carcinomas, suggesting a higher mutational load and/or a bias towards genes included in the panel compared to GEJ lesions. Although the absence of differences in actionable mutations suggests that tumors in these sites can be considered together, the differences in TP53 and Wnt component mutation rates support the recent push to use location to distinguish proximal and distal gastric carcinomas as separate entities. Based on gene expression data, Shah et al. recently suggested that gastric carcinomas be grouped into three different subtypes [16]. The detection of more frequent KRAS mutations within distal non-diffuse carcinomas in our dataset when using this subclassification further supports pathologic classification of gastric cancers based on location and histotype.

Overall, mutation frequencies within the targeted hotspots were detected at a similar rate as those observed with exome sequencing in the TCGA data, also suggesting that with appropriate design, panel sequencing could be a viable method for interrogating multiple genes with a single test. Cases with mutations in $\geq 3$ genes were also more common in the stomach in this cohort. However, the differences in mutation rates in TP53, KRAS, and APC/ $C T N N B 1$ between GEJ and gastric carcinomas were not observed within the TCGA cohort, even after comparing mutation frequencies within specific gastric locations. It is uncertain whether differences in case selection relating to etiology, geography or ethnicity could account for such differences, or whether differences in sequencing technology or bioinformatic analyses may also have contributed to these divergent observations. Further studies directly comparing the two approaches and comparing different patient populations will further enhance our understanding of GEJ and gastric carcinoma.

\section{Study limitations}

Regarding case selection, in the presence of gastroesophageal reflux many of the landmarks used to delineate the stomach from the esophagus are destroyed. This study relied on the epicenter of the tumor being $5 \mathrm{~cm}$ from the gastroesophageal junction. However, we derived this classification from pathology reports and could not confirm the gross descriptions, nor did we subclassify tumours by Siewert type. Many of the tumours in this series may have in fact been esophageal in origin, and this could explain the similarities of the tumours with 
esophageal adenocarcinoma (e.g. worse prognosis and rates of TP53 mutations). The patients' family histories were not recorded for correlation, and the presence of gastric and GEJ cancer risk factors such as Helicobacter infection and Barrett esophagus were also not recorded. Sampling for sequencing and tissue microarray construction was limited, and intratumoral heterogeneity was not addressed. No germline DNA was available for comparison; as a result some somatic variants, which contribute to carcinogenesis but are present at low frequencies as single nucleotide polymorphisms, may have been omitted. In addition, we did not perform validation with Sanger sequencing or other methods. As such, we could not confirm the assay's sensitivity and specificity on this series. The assay has been shown to be accurate in other studies and in our own laboratory. Further validation of this platform with Sanger sequencing or other methods would be required before this assay could be used clinically.

\section{Conclusions}

GEJ and gastric tumors differ in several clinicopathologic respects, including the frequencies of mutations in certain caner-related genes. Tailoring treatment towards individual gastric cancer patients will require in-depth characterization of their tumors. This study shows that such characterization will derive information from both traditional clinicopathologic parameters such as tumor location, as well as from emerging molecular assays. Targeted panel sequencing is an approach that can be applied towards routine pathology material and can simultaneously yield information on several genes. Refinement of this approach may be a powerful tool for pathologists and clinicians in the future.

\section{Additional files}

Additional file 1: Table S1. List of genes and sequences of primer pairs used for DNA amplicon library construction.

Additional file 2: Table S2. Clinicopathologic data for the 167 cases in this series, including site, stage, margin status, immunohistochemistry and survival.

Additional file 3: Table S3. Summary of the clinocopathologic variables in the cohort's clinicopathologic variables within proximal non-diffuse, diffuse, and distal non-diffuse carcinomas.

Additional file 4: Table S4. Univariate and multivariable analyses of clinicopathologic variables associated with progression-free survival. Univariate values were computed via the log-rank test, and multivariable values were computed via Cox Proportional Hazard regression analysis using forward stepwise selection.

Additional file 5: Table S5. Univariate and multivariable analyses of clinicopathologic variables associated with overall survival. Univariate values were computed via the log-rank test, and multivariable values were computed via Cox Proportional Hazard regression analysis using forward stepwise selection.

Additional file 6: Table S6. List of variant calls detected in each case in this series.
Additional file 7: Table S7. List of mutated genes in the TCGA gastric cancer dataset within regions that were amplified and sequenced in this study.

\section{Abbreviations}

GEJ: Gastroesophageal junction; FFPE: Formalin-fixed paraffin embedded; INDELs: Insertions/deletions; SNVs: Single nucleotide variants.

\section{Competing interests}

The authors declare that they have no competing interests.

\section{Authors' contributions}

HeLC, DFS, and SY drafted the manuscript. KK and SJ performed analysis of the TCGA data. AL, YN, and EK carried out the molecular genetic studies, sequence alignment, and variant calling. HoL coordinated the retrospective review of the clinical data. $\mathrm{DH}$ provided critical review of the manuscript. HeLC performed the statistical analysis. HoL and SY conceived of the study, and participated in its design and coordination and helped to draft the manuscript. All authors read and approved the final manuscript.

\section{Acknowledgements}

This work was supported by grants from the British Columbia (BC) Cancer Foundation, largely through a private donation from Mr. Lorne Wickerson. HL receives fellowship funding from the Terry Fox Foundation Strategic Health Research Training Program in Cancer Research at the Canadian Institute for Health Research. KK is funded by the Canadian Institute of Health Research.

\section{Author details}

'University of British Columbia, Vancouver, Canada. ${ }^{2}$ Division of Anatomic Pathology, Department of Pathology and Laboratory Medicine, Vancouver General Hospital, 85512 Ave W, Vancouver, BC V5Z 1 M9, Canada. ${ }^{3}$ Department of Molecular Oncology, British Columbia Cancer Agency, Vancouver, Canada. ${ }^{4}$ Canada's Michael Smith Genome Sciences Centre, British Columbia Cancer Agency, Vancouver, Canada. ${ }^{5}$ Centre for Translational and Applied Genomics, British Columbia Cancer Agency, Vancouver, Canada. ${ }^{6}$ Department of Medical Oncology, British Columbia Cancer Agency, Vancouver, Canada.

Received: 23 June 2014 Accepted: 14 January 2015

Published online: 06 February 2015

\section{References}

1. Jemal A, Bray F, Center MM, Ferlay J, Ward E, Forman D. Global cancer statistics. CA-Cancer J Clin. 2011;61:69-90.

2. Odze RD. Pathology of the gastroesophageal junction. Semin Diagn Pathol. 2005;22:256-65.

3. Hansen S, Vollset SE, Derakhshan MH, Fyfe V, Melby KK, Aase S, et al. Two distinct aetiologies of cardia cancer; evidence from premorbid serological markers of gastric atrophy and Helicobacter pylori status. Gut. 2007;56:918-25.

4. Derakhshan MH, Malekzadeh R, Watabe H, Yazdanbod A, Fyfe V, Kazemi A, et al. Combination of gastric atrophy, reflux symptoms and histological subtype indicates two distinct aetiologies of gastric cardia cancer. Gut. 2008;57:298-305

5. Ren J-S, Kamangar F, Qiao Y-L, Taylor PR, Liang H, Dawsey SM, et al. Serum pepsinogens and risk of gastric and oesophageal cancers in the General Population Nutrition Intervention Trial cohort. Gut. 2009;58:636-42.

6. Turati F, Tramacere I, La Vecchia C, Negri E. A meta-analysis of body mass index and esophageal and gastric cardia adenocarcinoma. Ann Oncol. 2013;24:609-17.

7. Buas MF, Vaughan TL. Epidemiology and risk factors for gastroesophageal junction tumors: understanding the rising incidence of this disease. Semin Radiat Oncol. 2013;23:3-9.

8. Huang Q, Shi J, Feng A, Fan X, Zhang L, Mashimo H, et al. Gastric cardiac carcinomas involving the esophagus are more adequately staged as gastric cancers by the 7th edition of the American Joint Commission on Cancer Staging System. Mod Pathol. 2011;24:138-46.

9. McColl KEL, Going JJ. Aetiology and classification of adenocarcinoma of the gastro-oesophageal junction/cardia. Gut. 2010;59:282-4.

10. Fléjou JF, Gratio V, Muzeau F, Hamelin R. p53 abnormalities in adenocarcinoma of the gastric cardia and antrum. Mol Pathol. 1999;52:263-8. 
11. Ireland AP, Shibata DK, Chandrasoma P. Clinical significance of p53 mutations in adenocarcinoma of the esophagus and cardia. Ann Surg. 2000;231:179-87

12. Sarbia M, Geddert H, Klump B, Kiel S, Iskender E, Gabbert HE. Hypermethylation of tumor suppressor genes (p16INK4A, p14ARF and APC) in adenocarcinomas of the upper gastrointestinal tract. Int J Cancer. 2004;111:224-8.

13. Tajima Y, Yamazaki K, Makino R, Nishino N, Masuda Y, Aoki S, et al. Differences in the histological findings, phenotypic marker expressions and genetic alterations between adenocarcinoma of the gastric cardia and distal stomach. Br J Cancer. 2007:96:631-8.

14. Xue L, Zhang X, Li Y, Yang H, Li X, Mi J, et al. Differences of immunophenotypic markers and signaling molecules between adenocarcinomas of gastric cardia and distal stomach. Hum Pathol. 2011:42:594-601.

15. Wang G, Hu N, Yang HH, Wang L, Su H, Wang C, et al. Comparison of global gene expression of gastric cardia and noncardia cancers from a high-risk population in China. PLoS One. 2013;8:e63826.

16. Shah MA, Khanin R, Tang L, Janjigian YY, Klimstra DS, Gerdes $H$, et al. Molecular classification of gastric cancer: a new paradigm. Clin Cancer Res. 2011;17:2693-701.

17. Bang Y-J, Van Cutsem E, Feyereislova A, Chung HC, Shen L, Sawaki A, et al, Trastuzumab in combination with chemotherapy versus chemotherapy alone for treatment of HER2-positive advanced gastric or gastro-oesophagea junction cancer (ToGA): a phase 3, open-label, randomised controlled trial. Lancet. 2010;376:687-97.

18. Pao W, Miller V, Zakowski M, Doherty J, Politi K, Sarkaria I, et al. EGF receptor gene mutations are common in lung cancers from "never smokers" and are associated with sensitivity of tumors to gefitinib and erlotinib. Proc Natl Acad Sci U S A. 2004;101:13306-112004

19. Pao W, Wang TY, Riely GJ, Miller VA, Pan Q, Ladanyi M, et al. KRAS mutations and primary resistance of lung adenocarcinomas to gefitinib or erlotinib. PLoS Med. 2005:2:e17.

20. Franklin WA, Haney J, Sugita M, Bemis L. KRAS mutation: comparison of testing methods and tissue sampling techniques in colon cancer. J Mol Diagn. 2010;12:43-50.

21. Beadling C, Neff TL, Heinrich MC, Rhodes K. Combining highly multiplexed PCR with semiconductor-based sequencing for rapid cancer genotyping. J Mol Diagn. 2013;15:171-6.

22. Kanehisa M, Goto S. KEGG: kyoto encyclopedia of genes and genomes. Nucleic Acids Res. 2000:28:27-30

23. Kanehisa M. Molecular network analysis of diseases and drugs in KEGG. Methods Mol Biol. 2013;939:263-75.

24. Benjamini $Y$, Hochberg $Y$. Controlling the false discovery rate: a practical and powerful approach to multiple testing. J R Stat Soc Ser B Stat Methodol. 1995:57:289-300

25. Janku F, Wheler JJ, Naing A, Falchook GS, Hong DS, Stepanek VM, et al. PIK3CA mutation h1047r is associated with response to PI3K/AKT/MTOR signaling pathway inhibitors in early-phase clinical trials. Cancer Res. 2013;73:276-84.

26. Bollag G, Tsai J, Zhang J, Zhang C, Ibrahim P, Nolop K, et al. Vemurafenib: the first drug approved for BRAF-mutant cancer. Nat Rev Drug Discov. 2012;11:873-86.

27. Herter-Sprie GS, Greulich H, Wong K-K. Activating mutations in ERBB2 and their impact on diagnostics and treatment. Front Oncol. 2013;3:86.

28. Lau C, Killian KJ, Samuels Y, Rudloff U. ERBB4 mutation analysis: emerging molecular target for melanoma treatment. Methods Mol Biol. 2014;1102:461-80

29. Gozgit JM, Wong MJ, Moran L, Wardwell S, Mohemmad QK, Narasimhan NI, et al. Ponatinib (AP24534), a multitargeted pan-FGFR inhibitor with activity in multiple FGFR-amplified or mutated cancer models. Mol Cancer Ther. 2012;11:690-9.

30. Liao RG, Jung J, Tchaicha J, Wilkerson MD, Sivachenko A, Beauchamp EM, et al. Inhibitor-sensitive FGFR2 and FGFR3 mutations in lung squamous cell carcinoma. Cancer Res. 2013;73:5195-205.

31. Koo GC, Tan SY, Tang T, Poon SL, Allen GE, Tan L, et al. Janus kinase 3-activating mutations identified in natural killer/T-cell lymphoma. Cancer Discov. 2012;2:591-7.

32. Gozgit JM, Wong MJ, Wardwell S, Tyner JW, Loriaux MM, Mohemmad QK, et al. Potent activity of ponatinib (AP24534) in models of FLT3-driven acute myeloid leukemia and other hematologic malignancies. Mol Cancer Ther. 2011;10:1028-35.
33. Chaturvedi A, Araujo Cruz MM, Jyotsana N, Sharma A, Yun H, Görlich K Mutant IDH1 promotes leukemogenesis in vivo and can be specifically targeted in human AML. Blood. 2013;122:2877-87.

34. Shah MA, Wainberg ZA, Catenacci DVT, Hochster HS, Ford J, Kunz P, et al. Phase II study evaluating 2 dosing schedules of oral foretinib (GSK1363089), cMETNEGFR2 inhibitor, in patients with metastatic gastric cancer. PLoS One. 2013;8:e54014

35. McTique M, Murray BW, Chen JH, Deng Y-L, Solowiej J, Kania RS. Molecular conformations, interactions, and properties associated with drug efficiency and clinical performance among VEGFR TK inhibitors. Proc Natl Acad Sci U S A. 2012;109:18281-9.

36. Jänne PA, Shaw AT, Pereira JR, Jeannin G, Vansteenkiste J, Barrios C, et al. Selumetinib plus docetaxel for KRAS-mutant advanced non-small-cell lung cancer: a randomised, multicentre, placebo-controlled, phase 2 study. Lancet Oncol. 2013;14:38-47.

37. Lee JH, Kim Y, Choi JW, Kim YS. Correlation of Imatinib resistance with the mutational status of KIT and PDGFRA genes in gastrointestinal stromal tumors: a meta-analysis. J Gastrointestin Liver Dis. 2013;22:413-8.

38. Yu B, Liu W, Yu W-M, Loh ML, Alter S, Guvench O, et al. Targeting protein tyrosine phosphatase SHP2 for the treatment of PTPN11-associated malignancies. Mol Cancer Ther. 2013;12:1738-48.

39. De Falco V, Buonocore P, Muthu M, Torregrossa L, Basolo F, Billaud M, et al. Ponatinib (AP24534) is a novel potent inhibitor of oncogenic RET mutants associated with thyroid cancer. J Clin Endocrinol Metab. 2013;98:E811-9.

40. Berlin J, Bendell JC, Hart LL, Firdaus I, Gore I, Hermann RC, et al. A randomized phase II trial of vismodegib versus placebo with FOLFOX or FOLFIRI and bevacizumab in patients with previously untreated metastatic colorectal cancer. Clin Cancer Res. 2013:19:258-67.

41. Dulak AM, Stojanov P, Peng S, Lawrence MS, Fox C, Stewart C, et al. Exome and whole-genome sequencing of esophageal adenocarcinoma identifies recurrent driver events and mutational complexity. Nat Genet. 2013;45:478-86.

42. Vogelstein B, Papadopoulos N, Velculescu VE, Zhou S, Diaz Jr LA, Kinzler KW. Cancer genome landscapes. Science. 2013;339:1546-58

43. Wadhwa R, Song S, Lee J-S, Yao Y, Wei Q, Ajani JA. Gastric cancer-molecular and clinical dimensions. Nat Rev Clin Oncol. 2013;10:643-55.

44. Muzny DM, Bainbridge MN, Chang K, Dinh HH, Drummond JA, Fowler G, et al. Comprehensive molecular characterization of human colon and rectal cancer. Nature. 2012:487:330-7.

45. Holbrook JD, Parker JS, Gallagher KT, Halsey WS, Hughes AM, Weigman VJ, et al. Clinical trial designs for evaluating the medical utility of prognostic and predictive biomarkers in oncology. J Transl Med. 2011;9:33-47.

46. Tsongalis GJ, Peterson JD, de Abreu FB, Tunkey CD, Gallagher TL, Strausbaugh LD, et al. Routine use of the Ion Torrent Ampliseq ${ }^{\text {TM }}$ Cancer Hotspot Panel for identification of clinically actionable somatic mutations. Clin Chem Lab Med. 2013;52:707-14.

47. Endris $V$, Penzel $R$, Warth A, Muckenhuber A, Schirmacher $P$, Stenzinger $A$, et al. Molecular diagnostic profiling of lung cancer specimens with a semiconductor-based massive parallel sequencing approach: feasibility, costs, and performance compared with conventional sequencing. J Mol Diagn. 2013;15:765-75

48. Singh RR, Patel KP, Routbort MJ, Reddy NG, Barkoh BA, Handal B, et al. Clinical validation of a next-generation sequencing screen for mutational hotspots in 46 cancer-related genes. J Mol Diagn. 2013;15:607-22.

49. Hadd AG, Houghton J, Choudhary A, Sah S, Chen L, Marko AC, et al. Targeted, high-depth, next-generation sequencing of cancer genes in formalin-fixed, paraffin-embedded and fine-needle aspiration tumor specimens. J Mol Diagn. 2013:15:234-47.

50. Kanagal-Shamanna R, Portier BP, Singh RR, Routbort MJ, Aldape KD, Handal $B A$, et al. Next-generation sequencing-based multi-gene mutation profiling of solid tumors using fine needle aspiration samples: promises and challenges for routine clinical diagnostics. Mod Pathol. 2013;27:314-27.

51. Scarpa A, Sikora K, Fassan M, Rachiglio AM, Cappellesso R, Antonello D, et al. Molecular typing of lung adenocarcinoma on cytological samples using a multigene next generation sequencing panel. PLoS One. 2013;8:e80478.

52. Nikiforova MN, Wald Al, Roy S, Durso MB, Nikiforov YE. Targeted next-generation sequencing panel (ThyroSeq) for detection of mutations in thyroid cancer. J Clin Endocrinol Metab. 2013;98:E1852-60.

53. Fassan M, Simbolo M, Bria E, Mafficini A, Pilotto S, Capelli $P$, et al. High-throughput mutation profiling identifies novel molecular dysregulation in high-grade intraepithelial neoplasia and early gastric cancers. Gastric Cancer. 2014;17:442-9. 
54. Soussi T, Roud CBE. Assessing TP53 status in human tumours to evaluate clinical outcome. Nat Rev Cancer. 2001;1:233-9.

55. Dang L, White DW, Gross S, Bennett BD, Bittinger MA, Driggers EM, et al. Cancer-associated IDH1 mutations produce 2-hydroxyglutarate. Nature. 2009;462:739-44.

56. Yanada M, Matsuo K, Suzuki T, Kiyoi H, Naoe T. Prognostic significance of FLT3 internal tandem duplication and tyrosine kinase domain mutations for acute myeloid leukemia: a meta-analysis. Leukemia. 2005;19:1345-9.

57. Matsuno N, Nanri T, Kawakita T, Mitsuya H, Asou N. A novel FLT3 activation loop mutation N841K in acute myeloblastic leukemia. Leukemia. 2005;19:480-1.

58. Walters DK, Mercher T, Gu T-L, O'Hare T, Tyner JW, Loriaux M, et al. Activating alleles of JAK3 in acute megakaryoblastic leukemia. Cancer Cell. 2006; $10: 65-75$.

59. Jeong EG, Kim MS, Nam HK, Min CK, Lee S. Somatic mutations of JAK1 and JAK3 in acute leukemias and solid cancers. Clin Cancer Res. 2008;14:3716-21.

60. Sharma P, Weston AP, Morales T, Topalovski M, Mayo MS, Sampliner RE. Relative risk of dysplasia for patients with intestinal metaplasia in the distal oesophagus and in the gastric cardia. Gut. 2000;46:9-13.

61. Islami F, Kamangar F. Helicobacter pylori and esophageal cancer risk: a meta-analysis. Cancer Prev Res (Phila). 2008;1:329-38.

62. Xie F-J, Zhang Y-P, Zheng Q-Q, Jin H-C, Wang F-L, Chen M, et al. Helicobacter pylori infection and esophageal cancer risk: an updated meta-analysis. World J Gastroenterol. 2013;19:6098-107.

\section{Submit your next manuscript to BioMed Central and take full advantage of:}

- Convenient online submission

- Thorough peer review

- No space constraints or color figure charges

- Immediate publication on acceptance

- Inclusion in PubMed, CAS, Scopus and Google Scholar

- Research which is freely available for redistribution 